\title{
EXTENDED AND CONDITIONAL VERSIONS OF THE PASTA PROPERTY
}

\author{
DIETER KÖNIG AND \\ VOLKER SCHMIDT, * Mining Academy of Freiberg
}

\begin{abstract}
Two types of conditions are discussed ensuring the equality between long-run time fractions and long-run event fractions of stochastic processes with embedded point processes. Modifications of this equality statement are considered.
\end{abstract}

\section{Introduction}

In the current literature (see the references below) several lines of investigation can be observed dealing with generalizations of the fact that Poisson Arrivals see time averages (PASTA). Roughly speaking, the methods used for proving such results can be divided into two classes: (i) Martingale techniques are exploited, where the embedded point process is assumed to admit a stochastic intensity. (ii) Conditional intensities are considered, including their local characterization in the sense of Korolyuk, where the existence of these conditional intensities is either assumed $a$ priori or ensured by the existence of a sufficiently rich family of regular subsets of the state space. We discuss these two approaches within the stationary framework. In particular, a general form of the conditional PASTA property is stated.

\section{The general stationary model}

Let $X=(X(t), t \in \mathscr{R})$ be a stochastic process on some probability space [ $\Omega, \mathscr{F}, P]$. The state space $J$ is an arbitrary complete separable metric space. $\mathscr{J}$ is the $\sigma$-algebra of its Borel sets. We assume that the trajectories of $X$ are left-continuous with right-hand limits. Furthermore, let $\Phi=\left(T_{n}, n \in \mathscr{Z}\right)$ be a point process in $\mathscr{R}$ with $\cdots\left\langle T_{0}<0 \leqq T_{1}<T_{2}<\cdots\right.$ which is also given on $[\Omega, \mathscr{F}, P] ; \mathscr{R}=(-\infty, \infty), \mathscr{Z}=\{\cdots,-1,0,1, \ldots\}$. By means of $X$ and $\Phi$ we define the marked point process $\Psi_{x}=\left(\left[T_{n},\left(X\left(T_{n}\right), X\left(T_{n}+0\right)\right)\right], n \in \mathscr{Z}\right)$ with the mark space $[J \times J, \mathscr{J} \otimes \mathscr{J}]$. The pair $\left[X, \Psi_{X}\right]$ we call a process with embedded marked point process (briefly: PMP). We assume that the PMP $\left[X, \Psi_{X}\right]$ is stationary, i.e. the processes $X$ and $\Psi_{X}$ are stationary, and they are stationarily connected.

\section{The martingale approach}

For extending the PASTA property by means of martingale techniques, the basic assumption is that $\Phi$ admits a stochastic intensity $(\lambda(t), t \in \mathscr{R})$. Then, under some additional assumptions, it holds (see Formula (4.3) in Brémaud [1] and Corollary 3 in Melamed and Whitt [6]) that

$$
\lambda^{-1} E(f(X(0)) \lambda(0))=E^{0}(f(X(0)))
$$

for every continuous bounded function $f: J \rightarrow \mathscr{R}$, where $E^{0}$ denotes the expectation taken with respect to the Palm distribution $P^{0}$ of $P ; \lambda=E \lambda(0)$. The formula (1) can be seen as an

Received 8 August 1989; revision received 28 November 1989.

* Postal address for both authors: Bergakademie Freiberg, Sektion Mathematik, Bernhard-von-CottaStr. 2, Freiburg, 9200, GDR. 
extended version of the PASTA property. Indeed, if $\Phi$ is Poisson it holds that $\lambda(t) \equiv \lambda$ and from (1) the equality

$$
P(X(0) \in A)=P^{0}(X(0) \in A) \text { for every } A \in \mathscr{J}
$$

follows. Moreover, in the general case (not assuming that $\Phi$ is Poisson) from (1) it follows (see Theorem 5.1 in [1] and Theorem 2 in [6]) that the distributions of $X(0)$ under $P$ and $P^{0}$ coincide iff

$$
E(\lambda(0) \mid X(0))=E \lambda(0)
$$

A discrete-time analogue of condition (3) has been derived in [5], where real-valued discrete-time stochastic processes with an embedded discrete-time point process are considered. We have looked for an example of PMP such that the processes $X$ and $\Phi$ are not independent, that $\Phi$ is non-Poisson admitting a stochastic intensity, and that nevertheless, (3) holds. But it seems to be difficult to construct such an example because under some mild additional assumptions it follows from (3) that $\Phi$ must be Poisson (see Theorem 3 in [6]). This type of result is usually called the anti-PASTA property.

On the other hand, the formula (1) can be used in establishing the following conditional version of the PASTA property. To do this we first assume that the distribution of $\lambda(0)$ is discrete. Then, from (1) we get

$$
\lambda^{-1} a P(X(0) \in A, \lambda(0)=a)=P^{0}(X(0) \in A, \lambda(0)=a)
$$

considering the vector process $\{(X(t), \lambda(t))\}$ and bearing in mind that by taking limits the validity of the formula (1) can be proved for arbitrary bounded measurable $f$. This gives

$$
P(X(0) \in A \mid \lambda(0)=a)=P^{0}(X(0) \in A \mid \lambda(0)=a) .
$$

In the case when $\Phi$ is a Cox process, the validity of (4) was proved in Regterschot and van Doorn [8], where (4) was called the conditional PASTA property. A related condition ensuring the validity of (4) has been established in König et al. [4] (see also König and Schmidt [3]).

With respect to a possible conditional anti-PASTA property we now have a different situation than in the anti-PASTA case mentioned above, because for the validity of (4) we need no special condition (such as (3) for (2)). Within the model considered, where the basic assumption is that $\Phi$ admits a stochastic intensity, (4) is always true.

If the distribution of $\lambda(0)$ is not discrete, the following relationship corresponding to (4) can be proved:

$$
\lim _{\varepsilon \downarrow 0} \mathscr{P}(X(0) \in A \mid-\varepsilon<\lambda(0)-a<\varepsilon)=\lim _{\varepsilon \downarrow 0} \mathscr{P}^{0}(X(0) \in A \mid-\varepsilon<\lambda(0)-a<\varepsilon)
$$

\section{Conditional intensities}

Another way of establishing conditions for the validity of (2) is given by certain conditional intensities. For this, instead of the assumption that the embedded point process $\Phi$ admits a stochastic intensity, in König and Schmidt [3] the notion of a regular subset of the state space is used assuming the existence of a sufficiently rich family $\mathscr{J}_{0} \subset \mathscr{J}$ of such regular sets. It is shown (see Theorem 5 in [3]) that for every $A \in \mathscr{J}_{0}$ with $P(X(0) \in A)>0$ the limit

$$
\lambda_{A}=\lim _{h \downarrow 0} \frac{1}{h} P\left(T_{1}<h \mid X(0) \in A\right)
$$

exists and

$$
P(X(0) \in A)-P^{0}(X(0) \in A)=P(X(0) \in A)\left(1-\frac{\lambda_{A}}{\lambda}\right)
$$


Similar to (1), the formula (6) can be seen as an extended version of the PASTA property. Namely, assuming that $X$ is adapted in some sense, in the Poisson case we have $\lambda_{A} \equiv \lambda$ and, consequently, (2) holds. When $\Phi$ is general, the equality (2) holds iff a subfamily $\mathscr{J}_{1} \subset \mathscr{J}_{0}$ of regular sets exists being closed under finite intersections and generating $\mathscr{J}$ such that

$$
\lambda_{A}=\lambda \quad \text { for every } A \in \mathscr{J}_{1} \text { with } P(X(0) \in A)>0
$$

(see Corollary 2 in [3]). Thus, in effect, (7) plays the role of condition (3) in the martingale approach.

Informally we can consider the $\operatorname{limit}_{\lim _{A \downarrow\{x\}}} \lambda_{A}=\lambda_{\{x\}}$ provided that it exists in some sense. Then the mapping $\lambda_{\{X(0)\}}: \Omega \rightarrow[0, \infty)$ can be identified with the limit appearing in Formula (16) of Melamed and Whitt [7]. However, apart from concrete examples, it seems to be difficult to prove the existence of $\lambda_{\{X(0)\}}$. Clearly, when $\lambda_{\{X(0)\}}$ exists, the condition (7) is equivalent to $\lambda_{\{x(0)\}}=\lambda$ which is used in [7]. Under the assumption that the state space $J$ is discrete, in Stidham and El Taha [9] a sample-path analogue of this condition and of formula (6) is obtained (see Theorem 3.5 and Corollary 3.6 in [9]).

Furthermore, in [3] a non-Poisson example is given such that (7) is satisfied although the processes $X$ and $\Phi$ are not independent (see Example 2 in [3]). Namely, the stationary single-server queue $G I / M / 1 / \infty$ is considered with the special interarrival time distribution investigated in König et al. [2]. The embedded points $T_{n}$ are assumed to be the departure epochs of customers. The process $X$ is the stationary virtual waiting time process. Then it is not difficult to show that (7) holds with $\mathscr{J}_{1}=\{(a, b): 0<a \leqq b<\infty\}$.

On the other hand, it seems not to be clear how to use the martingale approach for this example, i.e. how to prove the validity of condition (3). This contrasts with the fact that, with respect to the internal history of the marked input, the point process $\Phi$ of departure epochs admits a relatively elementary stochastic intensity $(\lambda(t), t \in \mathscr{R})$ given by $\lambda(t)=\lambda^{-}(t-0)$ with $\lambda^{-}(t)=\mu \mathbf{1}_{(0, \infty)}(X(t))$.

\section{References}

[1] BRÉmAud, P. (1989) Characteristics of queueing systems observed at events and the connection between stochastic intensity and Palm probability. Queueing Systems 5.

[2] König, D., MiYaZawa, M. AND Schmidt, V. (1983) On the identification of Poisson arrivals in queues with coinciding time-stationary and customer-stationary state distributions. J. Appl. Prob. 20, 860-871.

[3] KönIG, D. AND SCHMIDT, V. (1989) EPSTA. The coincidence of time-stationary and customer-stationary distributions. Queueing Systems 5, 247-264.

[4] König, D., SchmidT, V. AND VAN Doorn, E. A. (1989) On the 'PASTA' property and a further relationship between customer and time averages in stationary queueing systems. Stochastic Models $\mathbf{5}$, 261-272.

[5] Makowski, A., Melamed, B. And Whitt, W. (1989) On averages seen by arrivals in discrete time. Proc. 28th IEEE Conference on Decision and Control, Tampa, Florida.

[6] Melamed, B. AND WhitT, W. (1989) On arrivals that see time averages: A martingale approach. J. Appl. Prob. 26.

[7] Melamed, B. AND WhitT, W. (1990) On arrivals that see time averages. Operat. Res. 37.

[8] Regterschot, G. J. K. AND van Doorn, E. A. (1988) Conditional PASTA. Operat. Res. Letters 7, 229-232.

[9] Stidham, S., JR. AND El TAHA, M. (1989) Sample-path analysis of processes with embedded point processes. Queueing Systems 5. 